\title{
Atypical Skin Rash in Two Infants Suspected to have Multi System Inflammatory Syndrome in a Developing Country with Positive Covid-19 Cases: Challenges Encountered
}

\section{Chisom Nri-Ezedi ${ }^{1 *}$, Thomas Ulasi ${ }^{1}$, Promise Monday ${ }^{2}$, Ogochukwu C. Ofiaeli $^{1}$ and Arinze Ulasi ${ }^{3}$}

${ }^{1}$ Department of Paediatrics, Faculty of Medicine, Nnamdi Azikiwe University, Awka, Anambra, Nigeria.

${ }^{2}$ Mid and South Essex NHS Foundation Trust, Broomfield Hospital, Essex, United Kingdom

${ }^{3}$ Department of Paediatrics, Nnamdi Azikiwe University Teaching Hospital Nnewi, Anambra state, Nigeria

*Corresponding Author: Chisom Nri-Ezedi, Lecturer and Consultant, Department of Paediatrics, Nnamdi Azikiwe University Teaching Hospital, Nnewi, Anambra, Nigeria.
Received: July 04, 2020

Published: July 25, 2020

(C) All rights are reserved by Chisom

Nri-Ezedi., et al.

DOI: 10.31080/ASPE.2020.03.0273

\begin{abstract}
Introduction: Globally, unusual cases characterised by fever, shock and Kawasaki-like rash termed multisystem inflammatory syndrome (MIS) have recently been reported in children, in the midst of the ongoing COVID-19 pandemic. In keeping with WHO criteria and call for increased surveillance of this syndrome, we are reporting two cases of suspected multisystem inflammatory syndrome in infants resident in an area reported to have positive cases of COVID-19.

Case Summaries: Two infants referred to the emergency department of a tertiary hospital in Nigeria with complaints of fever, vomiting, diarrhoea, and skin rash. On clinical examination, both patients were in shock with an evolving hyperpigmented skin rash associated with peeling, desquamation and swelling of both hands and feet. These cases, at the time of presentation, resided in localities reported to have positive COVID-19 cases.

Conclusion: There is need for increased surveillance and awareness of this syndrome among healthcare providers who take care of children in this ongoing pandemic.

Keywords: COVID-19; Kawasaki; Multisystem Inflammatory Syndrome; MIS-C; Skin Rash; Corona Virus; Skin Rash; Pandemic
\end{abstract}

\section{Introduction}

In December 2019, a novel strain of corona virus responsible for high rate of severe pneumonia, was first discovered in Wuhan City, Hubei Province of China and has since evolved over succeeding months, to a global pandemic of varied symptomatology. As of June 2020, this virus has affected 10 million people and counting worldwide, and is responsible for half a million deaths mostly affecting the elderly and adults with an underlying history of noncommunicable diseases [1]. In Africa, the incidence of this viral disease is increasing with the most reported deaths in Nigeria [2]. Although prior cases in children has remained low globally, emerging data from countries in Europe and North America, have demonstrated an increase in an unusual pattern of clinical features in children characterised by fever, inflammation, and multiorgan failure [3-5]. This reaction termed multisystem inflammatory syndrome in children (MIS-C) is believed to be linked with COVID-19 due to positive serology and/or PCR results in affected cases. So far, several modalities proposed to control this pandemic include optimal viral testing, constant use of face masks especially in public spaces, good hand hygiene, social distancing and ultimately the development of vaccines. However, adopting these measures particularly that which entails behavioural modi- 
fication have proved quite difficult, hence slowing efforts aimed to curb the spread of the virus. To address these growing concerns of MIS-C, health agencies have developed several criteria to aid in the rapid detection and timely report of suspected cases $[6,7]$. Collation of these data is vital in determining the epidemiological pattern of MIS-C, associated risk factors and modalities of treatment. Our report provides details of two infants suspected to have MIS-C, having largely met the clinical parameters adopted in the preliminary case definition adopted by WHO.

\section{Case Presentation}

\section{Case 1}

On $6^{\text {th }}$ of May, 2020, a 10 month old female was referred from a Diocesan hospital in Umunze, Orumba South Local Government Area, Anambra state, Nigeria to the Emergency room of Nnamdi Azikiwe University Teaching Hospital, Nnewi with complaints of a week duration of diarrhoea, vomiting and fever, and a day history of convulsion and loss of consciousness. Further history revealed an abdominal distension, bloody stool, and anaemia, which had led to blood transfusion at the referral site. On examination, the child was unconscious, acutely ill-looking and in respiratory distress, febrile with a temperature of $40 \mathrm{C}$, plethoric with a reddish tongue, and with no palpable peripheral pulses or blood pressure reading. There was global hypotonia, hepatomegaly, absent abdominal sounds and widespread crepitations in the lungs. Weight was $8.7 \mathrm{~kg}$ which was within typical values. A presumptive diagnosis of severe sepsis with septic shock led initiation of anti-shock therapy, vasoactive agents and oxygen. Anti-convulsants (phenobarbitone) and broad-spectrum antibiotics (meronem and metronidazole) were also given. Investigations done revealed lymphocytosis, neutropenia, a packed cell volume of $0.53 \mathrm{~L} / \mathrm{L}$ (presumed to be responsible for the plethora and reddish tongue), monocytosis and raised BUN values. Caregivers were unable to afford other needed tests. A week into admission, the clinical state of the patient deteriorated with rapid progression of extensive polymorphous skin lesions characterised by hyperpigmented patches, swollen hands and feet (nonpitting), desquamation, peeling and sparsely distributed bullae. These lesions were localised at the groin, hands, feet, back, scalp, flanks and flexural areas. Initially, we had suspected staphylococcal scalded skin lesion, which was confirmed clinically by both the dermatologists and plastic surgeons invited to clean and dress the skin. Medications were changed to anti-staphylococcal agents (flucloxacillin and gentamicin) and skin dressings were com- menced. However, no improvement was noted as the skin lesions progressively worsened on the second week of admission resulting in sloughing off of $60 \%$ of the body surface area. With additional symptoms such as mild conjunctivitis, and with non-improvement of the clinical state, a Kawasaki-like syndrome was suspected in the background of MIS-C associated with COVID-19. Patient's caregivers were however unable to carry out further tests due to financial constraints. The team contacted the hospital's COVID-19 task force team for screening of the virus, however this was not done because the patient did not meet the nation's criteria for screening. On the $17^{\text {th }}$ day of admission, patient died, and caregivers consoled.

\section{Case 2}

Thirty days following the admission of Case I, an 11-month-old male from Okija, Ihiala LGA, Anambra was referred to Emergency room with 2 week history of diarrhoea, 9 days of fever, abdominal distention of 5 days and skin rash of 3 days. Prior to presentation, there was a positive history of blood transfusion in the referral hospital. On examination, patient was acutely ill-looking, febrile with a temperature of 38C, unconscious and in respiratory distress. Capillary refill was greater than 3 seconds, with a thread pulse. Abdomen was uniformly distended with decreased abdominal sounds. Chest was clinically clear with presence of an extensive, hyperpigmented peeling rash localised at the back of the trunk, flanks, groin, scalp and other flexural areas like axilla, neck, with sparsely distributed bullae, swollen hands and feet and desquamation (Figure 1). Mild non-suppurative conjunctivitis was present, tongue normal in colour and oral mucosa affected at the sides. Weight was 8.2 $\mathrm{kg}$ which was in keeping with average expected values. A working diagnosis of severe sepsis in shock with toxic epidermal necrolysis was made. Anti-shock therapy was commenced, alongside with antibiotics- ceftriaxone and metronidazole. Side laboratory investigations done indicated random blood glucose of $35 \mathrm{mg} / \mathrm{dl}$, packed cell volume of $0.28 \mathrm{~L} / \mathrm{L}$, with negative HIV test and rapid malaria test. Blood parameters showed neutrophila, lymphocytopenia and thrombocytopenia. Other investigations requested which included erythrocyte sedimentation rate, kidney profile test, blood culture amongst others were not done owing to financial constraints. On the second day of admission, patient's condition deteriorated with progressive spread of the rash, peeling and desquamation. An attempt to do a repeat random blood sugar with a glucometer led to the peeling of the entire left toe nail and skin. With these changes and non-response to therapy, multisystem inflammatory syndrome 


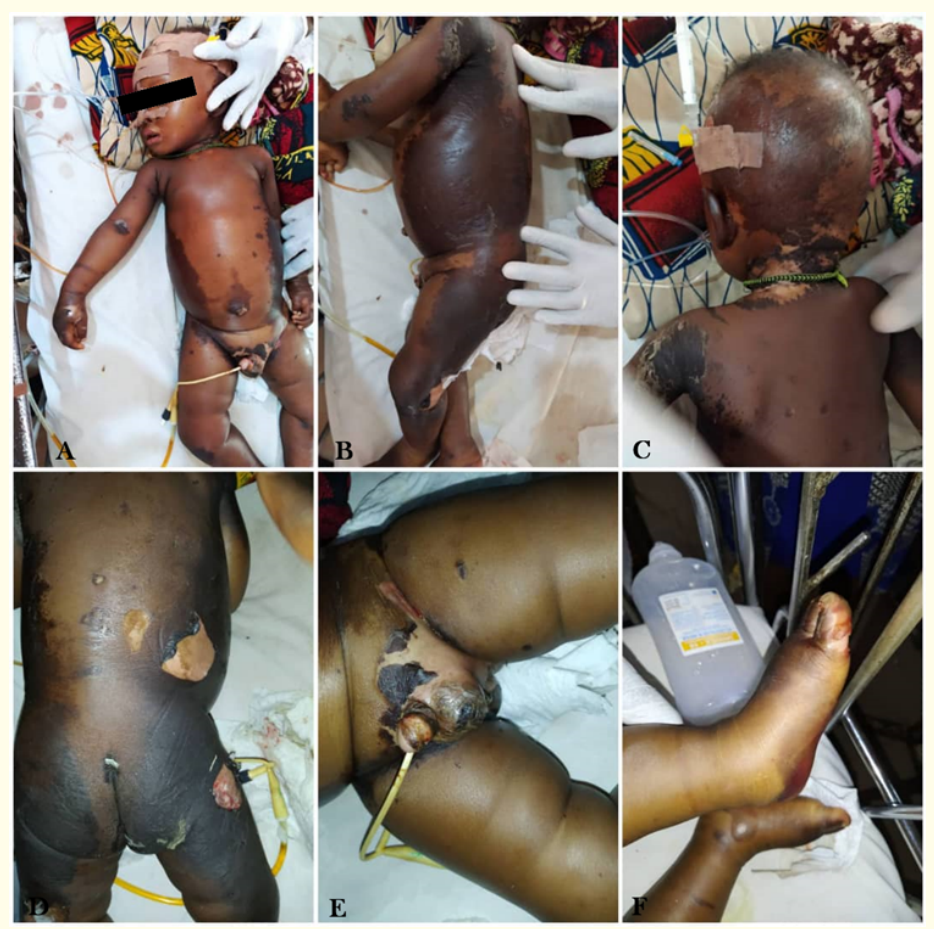

Figure 7: Case 2 with multiple extensive skin lesions: A- Anterior view depicting extensive patches at the flanks with peeling and bullae noted on the swollen hands; B- Better view of the hyperpigmented skin patch on the lateral body affecting also flexural areas and elbow;

C- Scalp, neck and axilla affected; D- Multiple sites of peeling with rash extending to the posterior lower limb; E- Groin also affected especially the flexural sites; F- Swollen feet with bullae, sole and desquamation of the left toes present.

associated with COVID-19 was suspected. Further tests requested were declined by the caregivers who insisted on being discharged against medical advice owing to their inability to meet up with financial health care demands and the lack of recovery after 48 hours of care. Comprehensive counselling was commenced and the social care workers invited to aid financially. We also invited plastic surgery and COVID-19 task force team for skin dressing, and screening respectively. Unfortunately, the viral test could not be conducted due to lack of available materials to collect the necessary samples. The next day, on the insistence of the caregivers, patient was discharged against medical advice.

\section{Discussion}

The report of two cases in our institution meeting the criteria adopted by WHO in the detection of MIS-C is not surprising owing to increasing reports of community spread in Nigeria. Whilst a lot of questions regarding the pathophysiology and management COVID-19 and MIS-C still remains largely unanswered, it has been theorized that the mechanism of MIS-C might be post infectious with an underlying immune disorder that activates the cytokine pathway. This hypothesis is mostly supported by the significant increase in positive serology testing of COVID-19 when compared to the proportion detected through reverse transcriptase polymerase chain reaction (RT-PCR) [3,5]. In a recently reported cases series involving 58 children in England, the average age diagnosed with MIS-C was 9 years (IQR: 5.7-14), which differs from the average age of 10.5 months observed in our report [3]. Race appears to be contributory factor as blacks and Hispanic children are disproportionately more affected compared to whites and Asians [8]. Using the case definition profile developed by health agencies globally, common presenting symptoms in MIS-C include persistent fever of at least 4 days, abdominal pain, vomiting, diarrhoea, rash, conjunctivitis, mucous membrane affectation, lethargy, confusion, swollen hands and feet, amongst others while common signs include shock, Kawasaki-like spectrum, and organ dysfunction [6,7]. Laboratory 
parameters show lymphocytopenia, neutrophilia, mild anaemia, thrombocytopenia, and elevated inflammatory and cardiac markers $[6,7]$. Majority of the clinical features as listed in criteria were present in the two cases that had presented in our facility: Persistent fever of at least 5 days, vomiting, diarrhoea, shock, altered consciousness, swollen hands and feet and polymorphous rash extensively comprising of an almost generalised sheet of thickened hyperpigmented/erythematous rash, and bullae associated with peeling, and desquamation. Similarly, the blood count parameters were partly present in case two.

In a recent report of MIS-C in patients aged 21 years and below in New York, dermatologic lesions were the leading symptoms in children aged 0-5 years [8]. The dramatic evolution of the skin rash in the two cases reported proved to be a diagnostic dilemma (Figure 1). An initial impression of Staphylococcal Scalded Skin Syndrome (SSSS) and its extended spectrum was made in both patients due to the presence of characteristic skin changes at the flexural areas, positive nikolsky sign, bullae, and desquamation. However, due to poor response to appropriate anti-staphylococcal antibiotics and evolving dermatologic lesions one of which includes swollen hands and feet- a different skin pathology was reconsidered. With the presence of other clinical features already previously highlighted in both patients but for some necessary laboratory investigations required to make a definitive diagnosis of Kawasaki-like syndrome, MIS-C was considered in view of the ongoing pandemic.

We had encountered several limitations in meeting up with the laboratory component of the WHO criteria. This is largely attributed to the poor socioeconomic status of the caregivers, and partly on the lack of available infrastructure, an ongoing dilemma exacerbated by the current pandemic and the fact that over $90 \%$ of health care funding is through out of pocket expenses with poor coverage of the national health insurance scheme. Although, efforts made to test for COVID-19 in both patients were unsuccessful as previously highlighted, there is strong evidence suggestive of a possible contact with a COVID-19 positive patient. Emerging data released by the Nigeria Centre for Disease Control (NCDC) demonstrate a significant increase in positive COVID-19 cases in Anambra in the preceding month with affected individuals residing in 15 out of 21 local government precincts [9]. In addition, the current national protocol dictates that only suspected cases should be tested for COVID-19 in designated centres approved by the NCDC, and if found positive, exposed contacts should be screened as well [9]. This practice precludes the testing of asymptomatic carriers which constitutes a majority of the populace. More so, among suspected cases, a significant proportion avoid the health facilities for fear of being stigmatized and isolated $[9,10]$. These factors, amongst others, not only fosters community spread but can skew data collation, thus curtailing efforts undertaken to curb the morbidity and mortality associated with COVID-19 in Nigeria. Despite these limitations, we believe that the two subjects met the criteria for MIS-C as adopted by WHO and in reporting these cases, we hope to contribute to global data on the existence of this emerging syndrome in our locality.

\section{Conclusion}

This report is a wake-up call for health care providers who take care of children as there is need for active surveillance of MIS-C and revision of the nation's screening criteria as it pertains to children. Adequate funding and robust testing in both adults and children remain a prerequisite aimed towards the mitigation of the morbidity and mortality associated with this virus.

\section{Bibliography}

1. Cheung, Eva W., et al. "Multisystem Inflammatory Syndrome Related to COVID-19 in Previously Healthy Children and Adolescents in New York City". JAMA - Journal of the American Medical Association, American Medical Association (2020).

2. Coetzee Bronwyné Jo’sean and Ashraf Kagee. "Structural Barriers to Adhering to Health Behaviours in the Context of the COVID-19 Crisis: Considerations for Low- and Middle-Income Countries". Global Public Health, Taylor and Francis (2020): 1-10.

3. Coronavirus (COVID-19) | WHO | Regional Office for Africa.

4. Coronavirus Disease 2019.

5. Dufort Elizabeth M., et al. "Multisystem Inflammatory Syndrome in Children in New York State". New England Journal of Medicine, Massachusetts Medical Society (2020): NEJMoa2021756.

6. HAN Archive - $00432 \mid$ Health Alert Network (HAN). 
7. Multisystem Inflammatory Syndrome in Children and Adolescents Temporally Related to COVID-19.

8. NCDC Coronavirus COVID-19 Microsite.

9. Verdoni Lucio., et al. "An Outbreak of Severe Kawasaki-like Disease at the Italian Epicentre of the SARS-CoV-2 Epidemic: An Observational Cohort Study". The Lancet 395 (2020): 1771-1778.

10. Whittaker Elizabeth., et al. "Clinical Characteristics of 58 Children with a Pediatric Inflammatory Multisystem Syndrome Temporally Associated with SARS-CoV-2". JAMA - Journal of the American Medical Association, American Medical Association (2020).

\section{Assets from publication with us}

- Prompt Acknowledgement after receiving the article

- Thorough Double blinded peer review

- Rapid Publication

- Issue of Publication Certificate

- High visibility of your Published work

Website: www.actascientific.com/

Submit Article: www.actascientific.com/submission.php

Email us: editor@actascientific.com

Contact us: +919182824667 